$\stackrel{N}{N}$

Global Journals Inc.

is
GLOBAL JOURNAL OF RESEARCHES IN ENGINEERING: F

ELECTRICAL AND ELECTRONICS ENGINEERING

Volume 18 Issue 2 Version 1.0 Year 2018

Type: Double Blind Peer Reviewed International Research Journal

Publisher: Global Journals

Online ISSN: 2249-4596 \& Print ISSN: 0975-5861

\title{
Low Probability of Intercept Frequency Hopping Signal Characterization Comparison using the Wigner Ville Distribution and the Choi Williams Distribution
}

By Daniel L. Stevens \& Stephanie A. Schuckers

Clarkson University Potsdam

Abstract- Low probability of intercept radar signals, which are often challenging to detect and characterize, have as their objective 'to see and not be seen'. Digital intercept receivers are currently moving from Fourier-based techniques to classical time-frequency techniques for the analysis of low probability of intercept radar signals. This paper presents the novel approach of characterizing low probability of intercept frequency hopping radar signals through utilization and direct comparison of the Wigner Ville Distribuion versus the Choi Williams Distribution. Two different frequency hopping low probability of intercept radar signals were analyzed (4-component and 8-component). The following metrics were used for evaluation: percent error of: carrier frequency, modulation bandwidth, modulation period, and time-frequency localization. Also used were: percent detection, lowest signaltonoise ratio for signal detection, and plot (processing) time.

Strictly as per the compliance and regulations of:

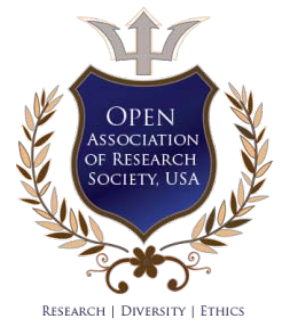

(C) 2018. Daniel L. Stevens \& Stephanie A. Schuckers. This is a research/review paper, distributed under the terms of the Creative Commons Attribution-Noncommercial 3.0 Unported License http://creativecommons.org/licenses/by-nc/3.0/), permitting all non commercial use, distribution, and reproduction in any medium, provided the original work is properly cited. 


\title{
Low Probability of Intercept Frequency Hopping Signal Characterization Comparison using the Wigner Ville Distribution and the Choi Williams Distribution ${ }^{1}$
}

\author{
Daniel L. Stevens ${ }^{\alpha} \&$ Stephanie A. Schuckers ${ }^{\sigma}$
}

Abstract- Low probability of intercept radar signals, which are often challenging to detect and characterize, have as their objective 'to see and not be seen'. Digital intercept receivers are currently moving from Fourier-based techniques to classical time-frequency techniques for the analysis of low probability of intercept radar signals. This paper presents the novel approach of characterizing low probability of intercept frequency hopping radar signals through utilization and direct comparison of the Wigner Ville Distribuion versus the Choi Williams Distribution. Two different frequency hopping low probability of intercept radar signals were analyzed (4-component and 8-component). The following metrics were used for evaluation: percent error of: carrier frequency, modulation bandwidth, modulation period, and time-frequency localization. Also used were: percent detection, lowest signalto-noise ratio for signal detection, and plot (processing) time. Experimental results demonstrate that overall, the Wigner Ville Distribution produced more accurate characterization metrics than the Choi Williams Distribution. An improvement in performance could potentially translate into saved equipment and lives.

\section{INTRODUCTION}

A low probability of intercept (LPI) radar that uses frequency hopping techniques changes the transmitting frequency in time over a wide bandwidth in order to prevent an intercept receiver from intercepting the waveform. The frequency slots used are chosen from a frequency hopping sequence, and this unknown sequence gives the radar the advantage over the intercept receiver in terms of processing gain. The frequency sequence appears random to the intercept receiver, therefore the possibility of it following the changes in frequency is remote [PAC09]. This prevents a jammer from jamming the transmitted

Author a: Air Force Research Laboratory Rome, NY 13441. e-mail: daniel.stevens.7@us.af.mil

Author $\sigma$ : Department of Electrical and Computer Engineering, Clarkson University Potsdam, NY 13699. e-mail: sschucke@clarkson.edu frequency [ADA04]. Frequency hopping radar performance depends only slightly on the code used, given that certain properties are met. This allows for a larger assortment of codes, making it more difficult to intercept.

Time-frequency signal analysis includes the analysis and processing of signals with time-varying frequency content. These signals are best represented by a time-frequency distribution [PAP94], [HAN00], which displays how the energy of the signal is distributed over the two-dimensional time-frequency plane [WEI03], [LIX08], [OZD03]. Processing of the signal may then exploit the features produced by the concentration of signal energy in two dimensions (time and frequency), vice one dimension (time or frequency) [BOA03], [LIY03]. Since noise has a tendency to spread out evenly over the time-frequency domain, while signals tend to concentrate their energies within limited time intervals and frequency bands; the local SNR of a noisy signal can be improved by using time-frequency analysis [XIA99]. In addition, the intercept receiver can increase its processing gain by implementing timefrequency signal analysis [GUL08].

Time-frequency distributions can be beneficial for the visual interpretation of signal dynamics [RAN01]. An experienced operator may be better able to detect a signal and extract its parameters by examining the timefrequency distribution [ANJ09].

\section{a) Wigner Ville Distribution (WVD)}

One of the most prominent members of the time-frequency analysis techniques family is the WVD. The WVD satisfies a large number of desirable mathematical properties. In particular, it is always realvalued, preserves time and frequency shifts, and satisfies marginal properties [AUG96], [QIA02]. The WVD, which is a transformation of a continuous time signal into the time-frequency domain, is computed by

\footnotetext{
${ }^{1}$ Approved for Public Release; Distribution Unlimited: Case Number 88ABW-2018-1360 20180319
} 
correlating the signal with a time and frequency translated version of itself, making it bilinear. The WVD exhibits the highest signal energy concentration in the time-frequency plane [WIL06]. By using the WVD, an intercept receiver can come close to having a processing gain near the LPI radar's matched filter processing gain [PAC09]. The WVD also contains cross term interference between every pair of signal components, which may limit its applications [GUL07], [STE96], and which can make the WVD time-frequency representation hard to read, especially if the components are numerous or close to each other, and the more so in the presence of noise [BOA03]. This lack of readability can in turn translate into decreased signal detection and parameter extraction metrics, potentially placing the intercept receiver signal analyst in harm's way.

The WVD of a signal $x(t)$ is given in equation (1) as:

$$
W_{x}(t, f)=\int_{-\infty}^{+\infty} x\left(t+\frac{\tau}{2}\right) x^{*}\left(t-\frac{\tau}{2}\right) e^{-j 2 \pi f \tau} d \tau
$$

or equivalently in equation (2) as:

$$
W_{x}(t, f)=\int_{-\infty}^{+\infty} X\left(f+\frac{\xi}{2}\right) X^{*}\left(f-\frac{\xi}{2}\right) e^{j 2 \pi \xi t} d \xi
$$

\section{b) Choi Williams Distribution (CWD)}

The CWD is a member of the Cohen's class of time-frequency distributions which use smoothing kernels [GUL07] to help reduce cross-term interference so prevalent in the WVD [BOA03], [PAC09], [UPP08]. The reduction in cross-term interference can make the time-frequency representation more readable and can make signal detection and parameter extraction more accurate. The down-side is that the CWD, like all members of Cohen's class, is faced with an inevitable trade-off between cross-term reduction and timefrequency localization. Because of this, the signal detection and parameter extraction benefits gained by the cross-term reduction may be offset by the decrease in time-frequency localization (smearing or widening of the signal).

The CWD of a signal $x(s)$ is given in equation (3) as:

$$
C W_{x}(t, f)=\sqrt{\frac{2}{\pi}} \iint_{-\infty}^{+\infty} \frac{\sigma}{|\tau|} e^{-2 \sigma^{2}(s-t)^{2} / \tau^{2}} x\left(s+\frac{\tau}{2}\right) x^{*}\left(s-\frac{\tau}{2}\right) e^{-j 2 \pi f \tau} d s d \tau
$$

As can be seen from equation (3), the CWD uses an exponential kernel in the generalized class of bilinear time-frequency distributions. Choi and Williams introduced one of the earliest 'new' distributions [CHO89], which they called the Exponential Distribution or ED. This new distribution overcomes several drawbacks of the Spectrogram and the WVD, providing decent localization with suppressed interferences [WIL92], [GUL07], [UPP08]. Interference terms tend to lie away from the axes in the ambiguity plane, while auto terms (signals) tend to lie on the axes. The Spectrogram kernel attenuates everything away from the $(0,0)$ point, the WVD kernel passes everything, and the CWD kernel passes everything on the axes and attenuates away from the axes. Thus, the CWD generally attenuates interference terms [PAC09], [HLA92]. This provides its reduced interference characteristic. The Spectrogram reduces interference also, but at a cost to the signal concentration.

\section{il. Methodology}

The methodologies detailed in this section describe the processes involved in obtaining and comparing metrics between the classical time-frequency analysis techniques of the Wigner Ville Distribution and the Choi Williams Distribution for the detection and characterization of low probability of intercept frequency hopping radar signals.

The tools used for this testing were: MATLAB (version 7.12), Signal Processing Toolbox (version 6.15), Wavelet Toolbox (version 4.7), Image Processing Toolbox (version 7.2), Time - Frequency Toolbox (version 1.0)

Testing (which was accomplished on a desktop computer) was performed for 2 different waveforms (4 component frequency hopping, 8 component frequency hopping). For each waveform, parameters were chosen for academic validation of signal processing techniques. Due to computer processing resources they were not meant to represent real-world values. The number of samples for each test was chosen to be 512, which seemed to be the optimum size for the desktop computer. Testing was performed at three different SNR levels: $10 \mathrm{~dB}, 0 \mathrm{~dB}$, and the lowest SNR at which the signal could be detected. The noise added was white Gaussian noise, which best reflects the thermal noise present in the IF section of an intercept receiver [PAC09]. Kaiser windowing was used, when windowing was applicable. 50 runs were performed for each test, for statistical purposes. The plots included in this paper were done at a threshold of $5 \%$ of the maximum intensity and were linear scale (not $\mathrm{dB}$ ) of analytic 
(complex) signals; the color bar represents intensity. The signal processing tools used for each task were the Wigner Ville Distribution and the Choi Williams Distribution.

Task 1 consisted of analyzing a frequency hopping (prevalent in the LPI arena [AMS09]) 4component signal whose parameters were: sampling frequency $=5 \mathrm{KHz}$; carrier frequencies $=1 \mathrm{KHz}, 1.75 \mathrm{KHz}$, $0.75 \mathrm{KHz}, \quad 1.25 \mathrm{KHz}$; modulation bandwidth $=1 \mathrm{KHz}$; modulation period $=.025 \mathrm{sec}$.

Task 2 was similar to Task 1 , but for a frequency hopping 8-component signal, whose parameters were: sampling frequency $=5 \mathrm{KHz}$; carrier frequencies $=$ $1.5 \mathrm{KHz}, 1 \mathrm{KHz}, 1.25 \mathrm{KHz}, 1.5 \mathrm{KHz}, 1.75 \mathrm{KHz}, 1.25 \mathrm{KHz}$, $0.75 \mathrm{KHz}, \quad 1 \mathrm{KHz} ; \quad$ modulation bandwidth $=1 \mathrm{KHz}$; modulation period $=.0125 \mathrm{sec}$.
After each particular run of each test, metrics were extracted from the time-frequency representation. The different metrics extracted were as follows:

1) Plot (processing) time: Time required for plot to be displayed.

2) Percent detection: Percent of time signal was detected - signal was declared a detection if any portion of each of the signal components (4 or 8 signal components) exceeded a set threshold (a certain percentage of the maximum intensity of the time-frequency representation).

Threshold percentages were determined based on visual detections of low SNR signals (lowest SNR at which the signal could be visually detected in the timefrequency representation) (see Figure 1).

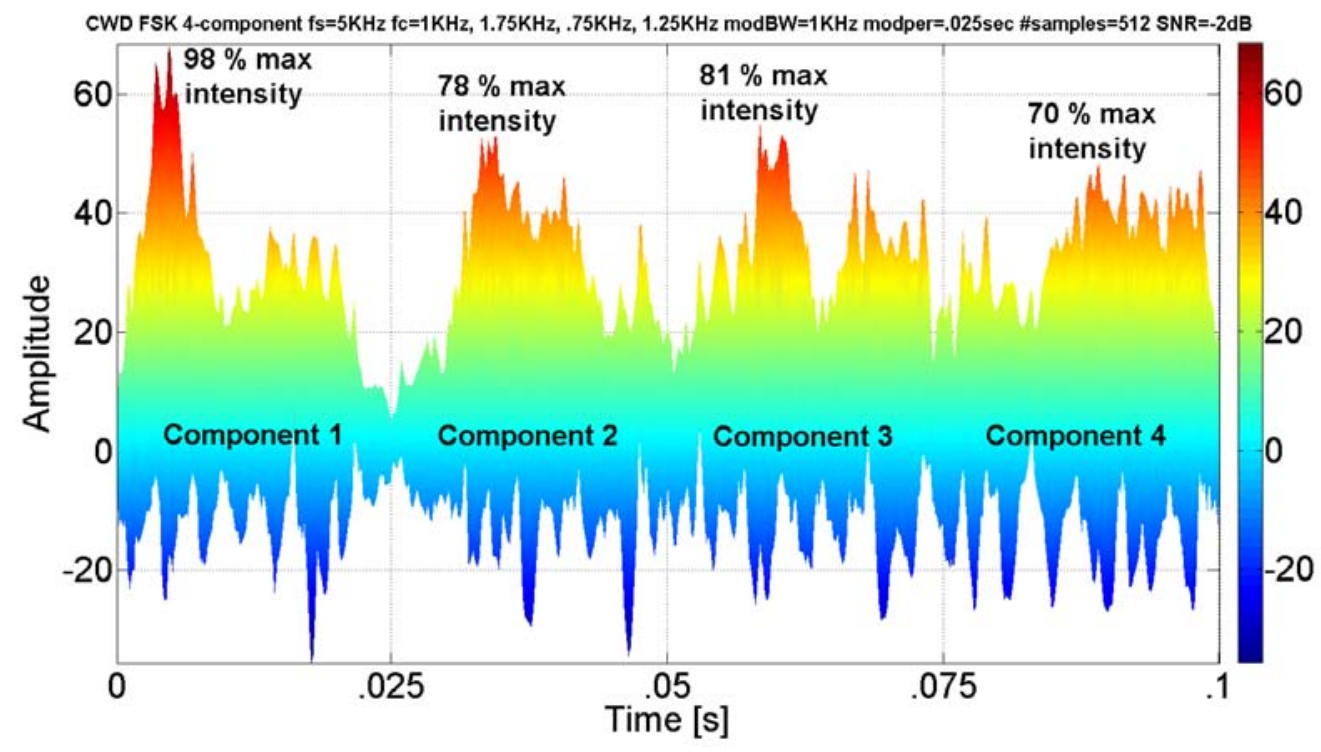

Figure 1: Threshold percentage determination. This plot is an amplitude vs. time ( $\mathrm{x}-\mathrm{z}$ view) of the CWD of a 4component frequency hopping signal (512 samples, $S N R=-2 d B$ ). For visually detected low SNR plots (like this one), the percent of max intensity for the peak z-value of each of the signal components was noted (here $98 \%, 78 \%$, $81 \%, 70 \%)$, and the lowest of these 4 values was recorded (70\%). Ten test runs were performed for this timefrequency analysis tool (CWD) for this waveform. The average of these recorded low values was determined and then assigned as the threshold for that particular time-frequency analysis tool. Note-the threshold for the CWD is $70 \%$.

Thresholds were assigned as follows: CWD (70\%); WVD (4-component FSK) (50\%); WVD (8-component FSK) (20\%)

For percent detection determination, these threshold values were included in the time-frequency plot algorithms so that the thresholds could be applied automatically during the plotting process. From the threshold plot, the signal was declared a detection if any portion of each of the signal components was visible (see Figure 2). 


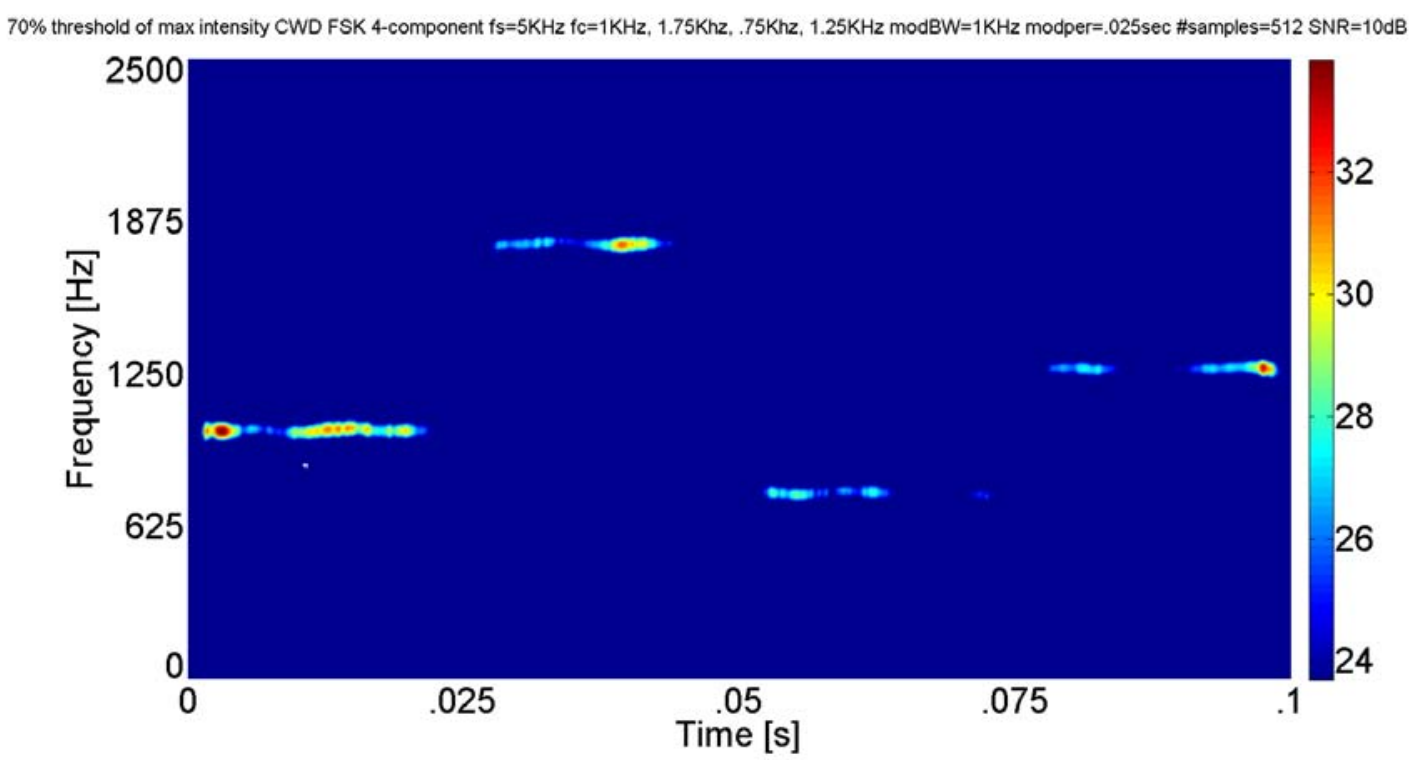

Figure 2: Percent detection (time-frequency). CWD of 4-component frequency hopping signal (512 samples, $\mathrm{SNR}=10 \mathrm{~dB}$ ) with threshold value automatically set to $70 \%$. From this threshold plot, the signal was declared a (visual) detection because at least a portion of each of the 4 FSK signal components was visible.

3) Carrier frequency: The frequency corresponding to the maximum intensity of the time-frequency representation (there are multiple carrier frequencies (4 or 8 ) for the frequency hopping waveforms).

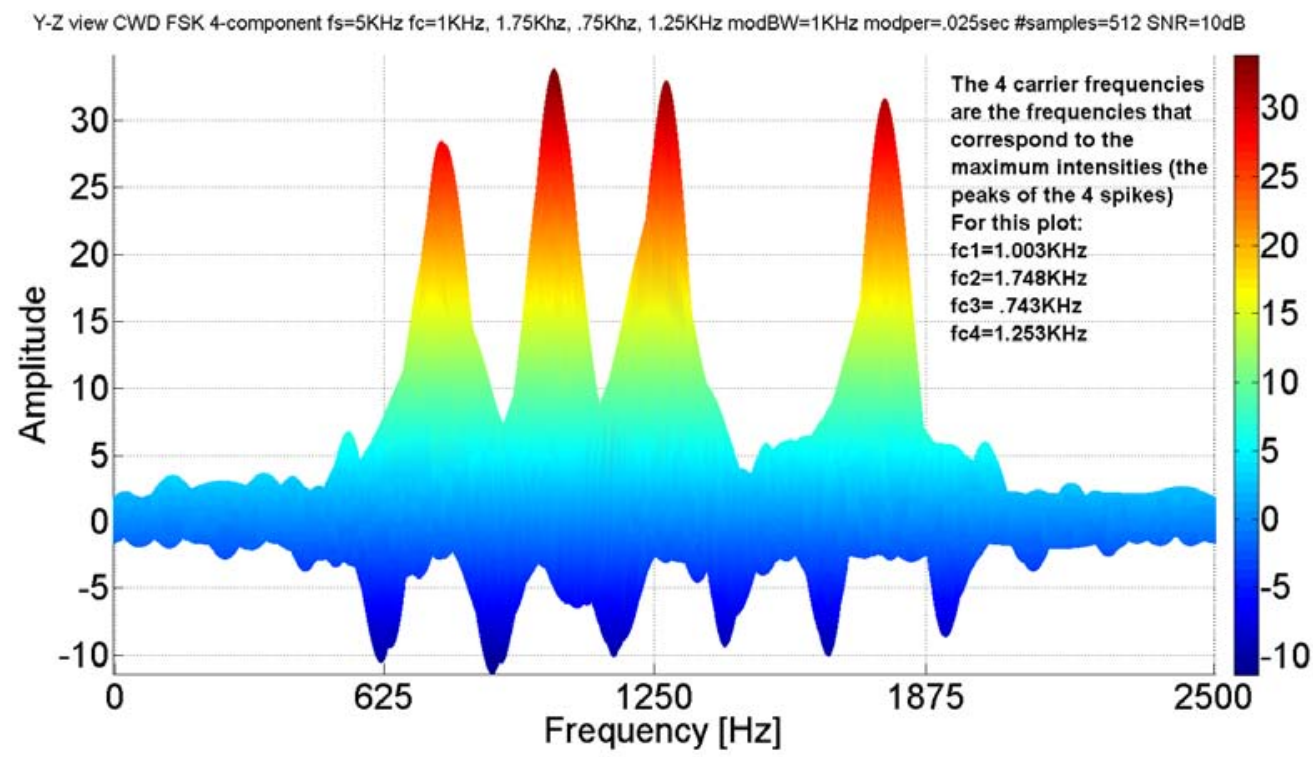

Figure 3: Determination of carrier frequency. CWD of a 4-component frequency hopping signal (512 samples, $\mathrm{SNR}=10 \mathrm{~dB}$ ). From the frequency-intensity $(\mathrm{y}-\mathrm{z})$ view, the 4 maximum intensity values ( 1 for each carrier frequency) are manually determined. The frequencies corresponding to those 4 max intensity values are the 4 carrier frequencies (for this plot fc $1=1003 \mathrm{~Hz}, \mathrm{fc} 2=1748 \mathrm{~Hz}$, fc3 $=743 \mathrm{~Hz}, \mathrm{fc} 4=1253 \mathrm{~Hz}$ ).

4) Modulation bandwidth: Distance from highest frequency value of signal (at a threshold of $20 \%$ maximum intensity) to lowest frequency value of signal (at same threshold) in Y-direction (frequency).

The threshold percentage was determined based on manual measurement of the modulation bandwidth of the signal in the time-frequency representation. This was accomplished for ten test runs of each time-frequency analysis tool (CWD and WVD), for each of the 2 waveforms. During each manual measurement, the max intensity of the high and low measuring points was recorded. The average of the 
max intensity values for these test runs was $20 \%$. This was adopted as the threshold value, and is representative of what is obtained when performing manual measurements. This $20 \%$ threshold was also adapted for determining the modulation period and the time-frequency localization (both are described below).
For modulation bandwidth determination, the $20 \%$ threshold value was included in the time-frequency plot algorithms so that the threshold could be applied automatically during the plotting process. From the threshold plot, the modulation bandwidth was manually measured (see Figure 4).

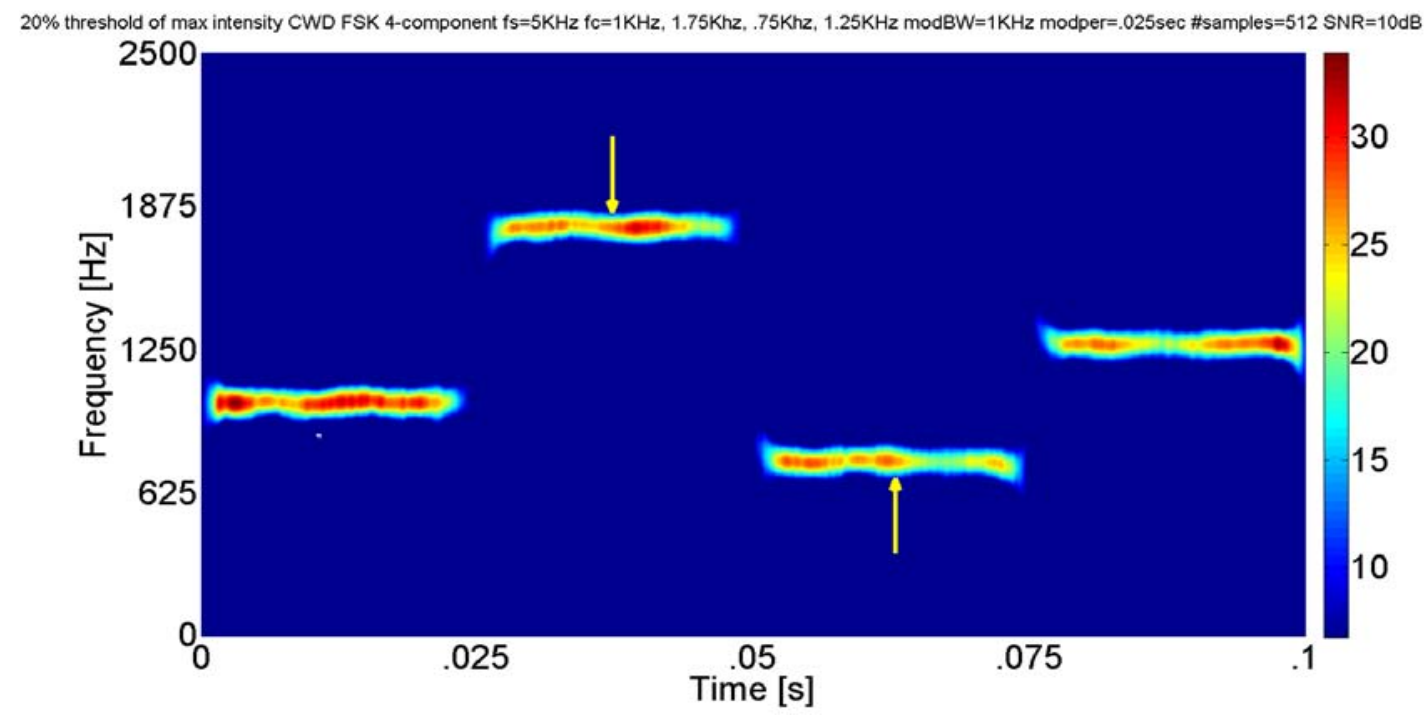

Figure 4: Modulation bandwidth determination. CWD of a 4-component frequency hopping signal (512 samples, $S N R=10 \mathrm{~dB}$ ) with threshold value automatically set to $20 \%$. From this threshold plot, the modulation bandwidth was measured manually from the highest frequency value of the signal (top yellow arrow) to the lowest frequency value of the signal (bottom yellow arrow) in the y-direction (frequency).

5) Modulation period: From Figure 5 (which is at a threshold of $20 \%$ maximum intensity), the modulation period is the manual measurement of the width of each of the 4 frequency hopping signals in the $x$-direction (time), and then the average of the 4 signals is calculated.

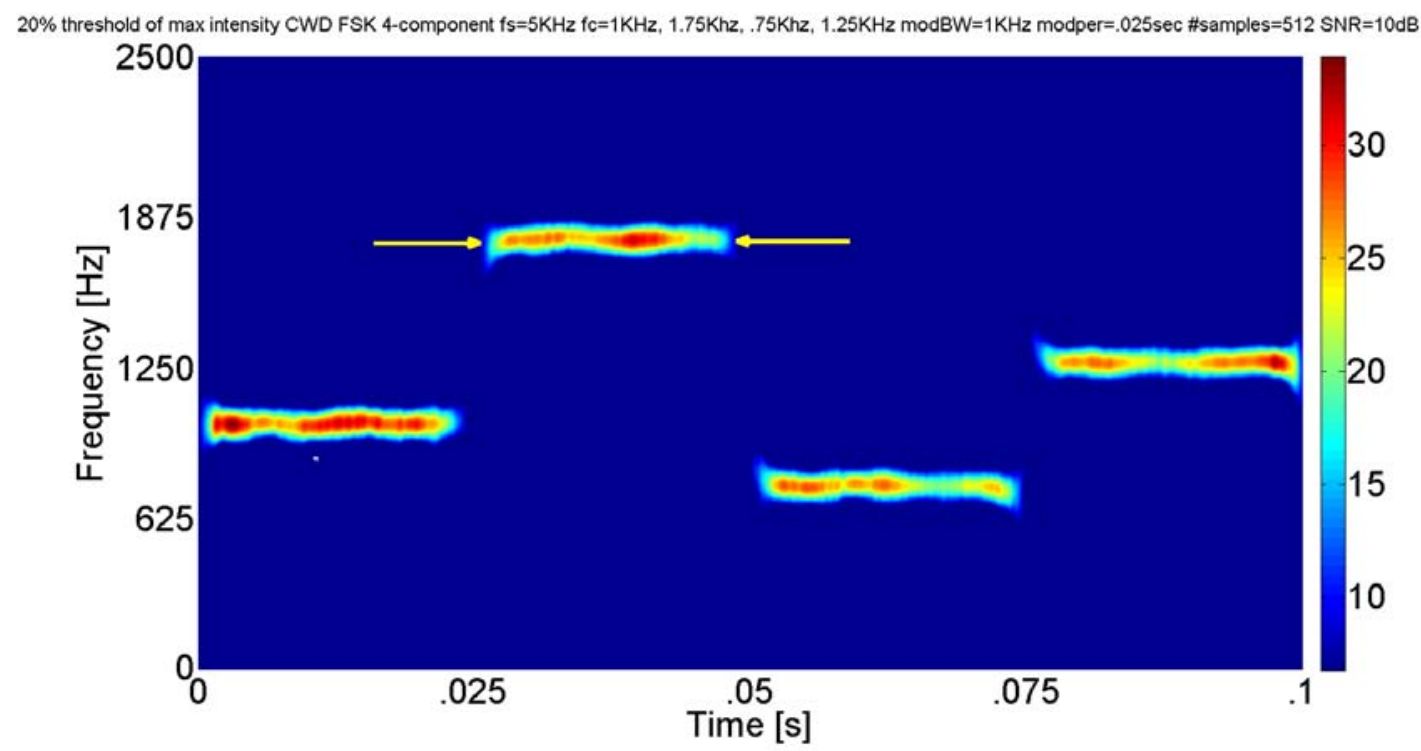

Figure 5: Modulation period determination. CWD of a 4-component frequency hopping signal (512 samples, $S N R=10 \mathrm{~dB}$ ) with threshold value automatically set to $20 \%$. From this threshold plot, the modulation period was measured manually from the left side of the signal (left yellow arrow) to the right side of the signal (right yellow arrow) in the $\mathrm{x}$-direction (time). This was done for all 4 signal components, and the average value was determined. 
6) Time-frequency localization: From Figure 6, the time-frequency localization is a manual measurement (at a threshold of $20 \%$ maximum intensity) of the 'thickness' (in the y-direction) of the center of each of the 4 frequency hopping signal components, and then the average of the 4 values are determined. The average frequency 'thickness' is then converted to: percent of the entire y-axis.

$20 \%$ threshold of max intensity CWD FSK 4-component fs $=5 \mathrm{KHz}$ fc $=1 \mathrm{KHz}, 1.75 \mathrm{Khz}, .75 \mathrm{Khz}, 1.25 \mathrm{KHz}$ modBW=1KHz modper=.025sec \#samples $=512 \mathrm{SNR}=10 \mathrm{~dB}$

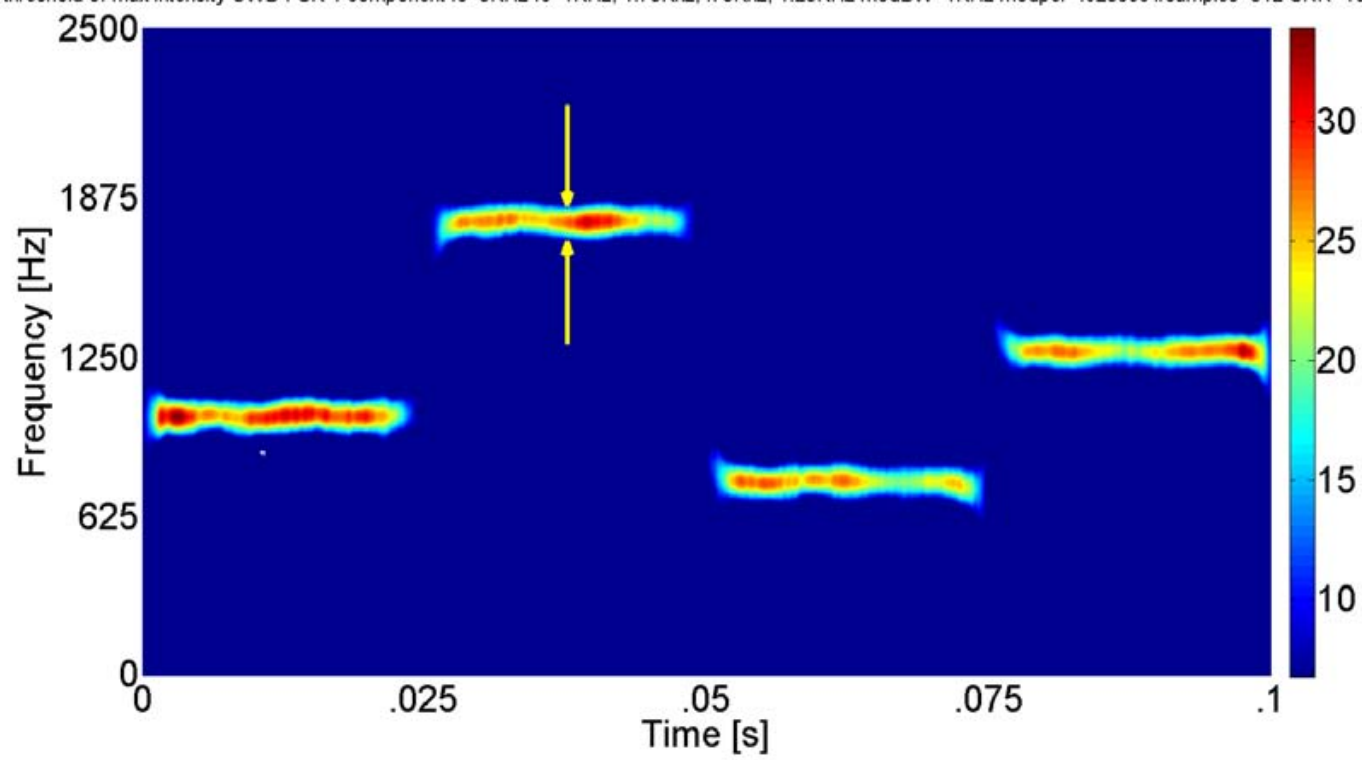

Figure 6: Time-frequency localization determination for the CWD of a 4-component frequency hopping signal (512 samples, $S N R=10 \mathrm{~dB}$ ) with threshold value automatically set to $20 \%$. From this threshold plot, the time-frequency localization was measured manually from the top of the signal (top yellow arrow) to the bottom of the signal (bottom yellow arrow) in the y-direction (frequency). This frequency 'thickness' value was then converted to: \% of entire y-axis.

7) Lowest detectable SNR: The lowest SNR level at which at least a portion of each of the signal components exceeded the set threshold listed in the percent detection section above.

For lowest detectable SNR determination, these threshold values were included in the time-frequency plot algorithms so that the thresholds could be applied automatically during the plotting process. From the threshold plot, the signal was declared a detection if any portion of each of the signal components was visible. The lowest SNR level for which the signal was declared a detection is the lowest detectable SNR (see Figure 7). 


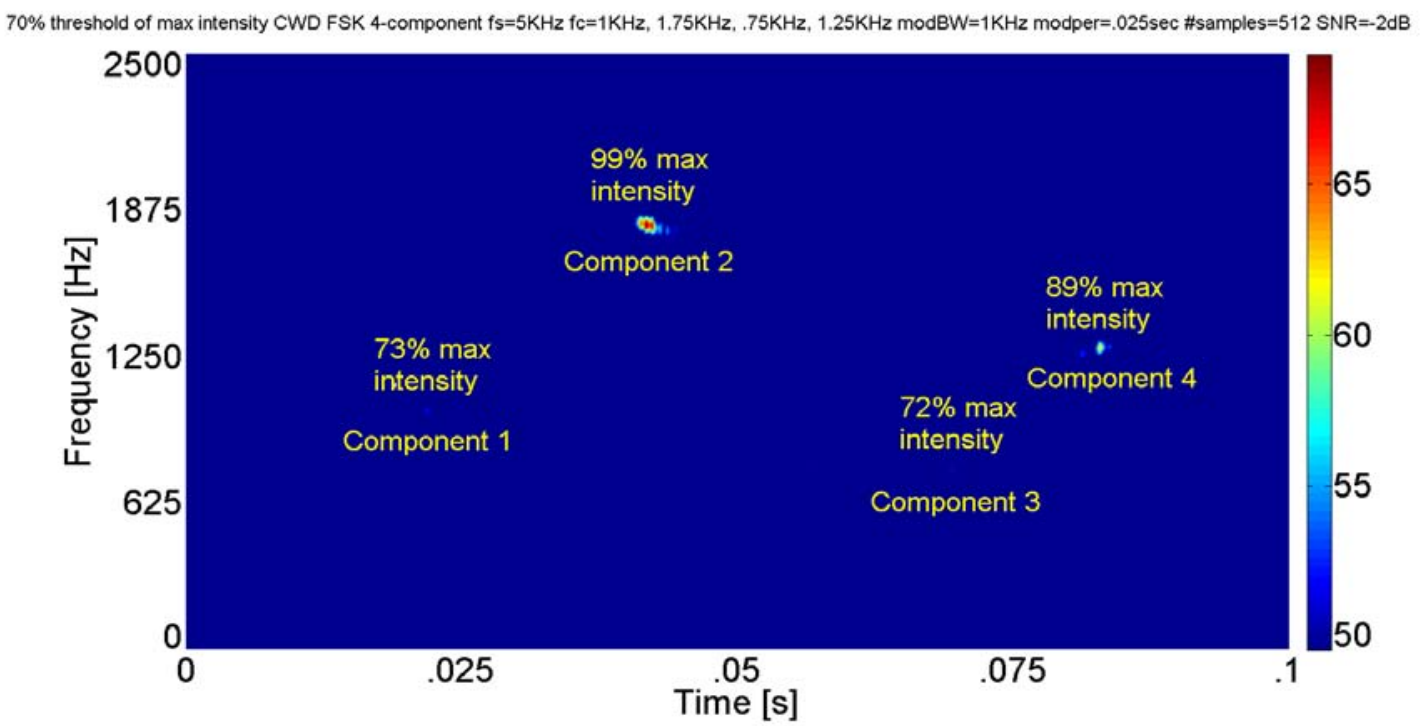

Figure 7: Lowest detectable SNR (time-frequency). CWD of 4-component frequency hopping signal (512 samples, $\mathrm{SNR}=-2 \mathrm{~dB}$ ) with threshold value automatically set to $70 \%$. From this threshold plot, the signal was declared a (visual) detection because at least a portion of each of the 4 frequency hopping signal components was visible. Note that the signal portion for the $73 \%$ max intensity and the $72 \%$ max intensity (just below the ' $n$ ' in 'intensity' for each case) is barely visible because the threshold for the CWD is 70\%. For this case, just a slightly lower SNR would have been a non-detect. Compare to Figure 2, which is the same plot, except that it has an SNR level equal to $10 \mathrm{~dB}$.

The data from all 50 runs for each test was used to produce the actual, error, and percent error for each of these metrics listed above.

The metrics from the WVD were then compared to the metrics from the CWD. By and large, the WVD outperformed the CWD, as will be shown in the results section.

Table 1: Overall test metrics (average percent error: carrier frequency, modulation bandwidth, modulation period; average: time-frequency localization-y (as percent of y-axis), percent detection, lowest detectable SNR, plot time) for the two classical time-frequency analysis techniques (WVD versus CWD).

\begin{tabular}{|c|c|c|}
\hline Parameters & WVD & CWD \\
\hline carrier frequency & $0.19 \%$ & $0.62 \%$ \\
\hline modulation bandwidth & $5.97 \%$ & $17.92 \%$ \\
\hline modulation period & $17.01 \%$ & $17.05 \%$ \\
\hline time-frequency localization-y & $2.04 \%$ & $6.78 \%$ \\
\hline percent detection & $90.7 \%$ & $88.7 \%$ \\
\hline lowest detectable SNR & $-2.0 \mathrm{db}$ & $-2.2 \mathrm{db}$ \\
\hline plottime & $6382 \mathrm{~s}$ & $10.16 \mathrm{~s}$ \\
\hline
\end{tabular}

From Table 1, the WVD outperformed the CWD in average percent error: carrier frequency $(0.19 \%$ vs. $0.62 \%)$, modulation bandwidth $(5.97 \%$ vs. $17.92 \%)$,

\section{Results}

Table 1 presents the overall test metrics for the two classical time-frequency analysis techniques used in this testing (WVD versus CWD). modulation period $(17.01 \%$ vs. $17.05 \%)$, and timefrequency localization (y-direction) $(2.04 \%$ vs. $6.78 \%$ ); and in average: percent detection (90.7\% vs. 
88.7\%), while the CWD outperformed the WVD in lowest detectable SNR (-2.2db vs. -2.0db) and average plot time (10.16s vs. 6382s).
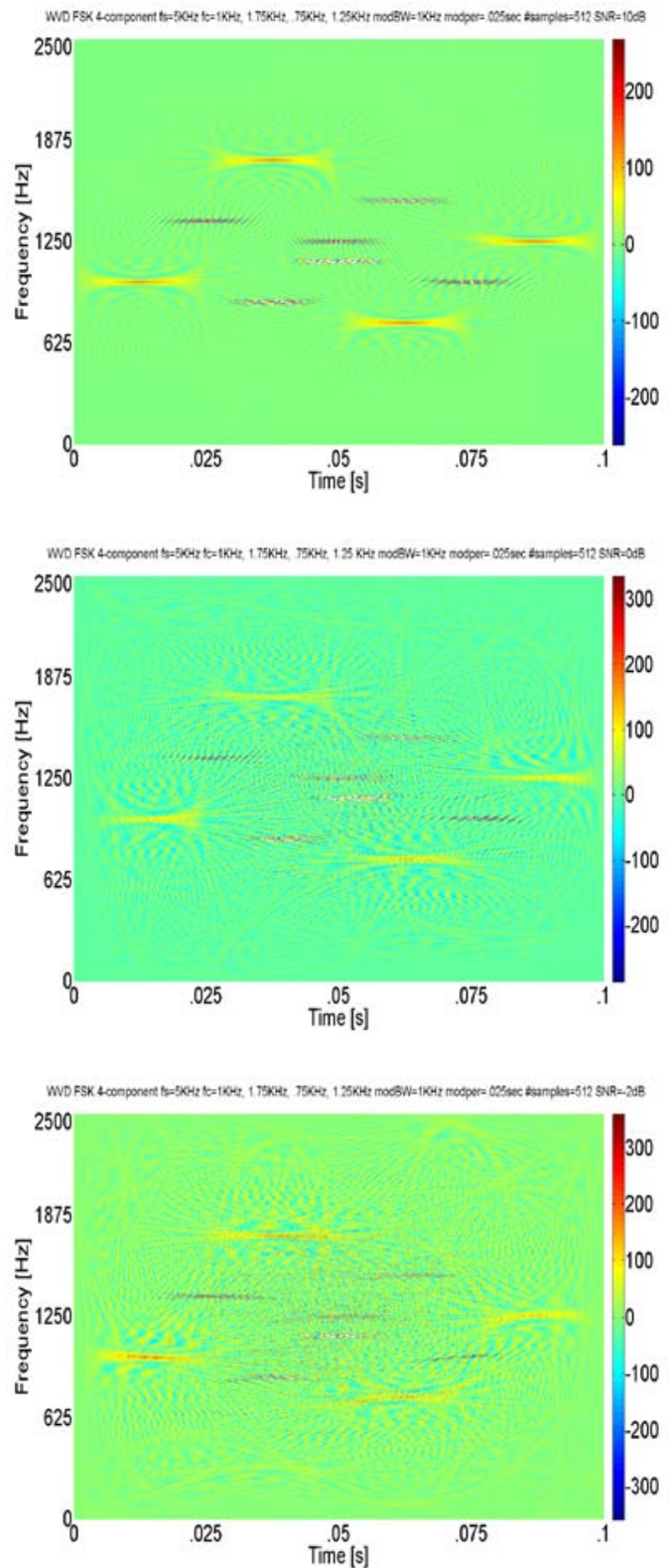

Figure 8 shows comparative plots of the WVD vs. the CWD (4 component frequency hopping) at SNRs of 10dB (top), OdB (middle), and -2dB (bottom).
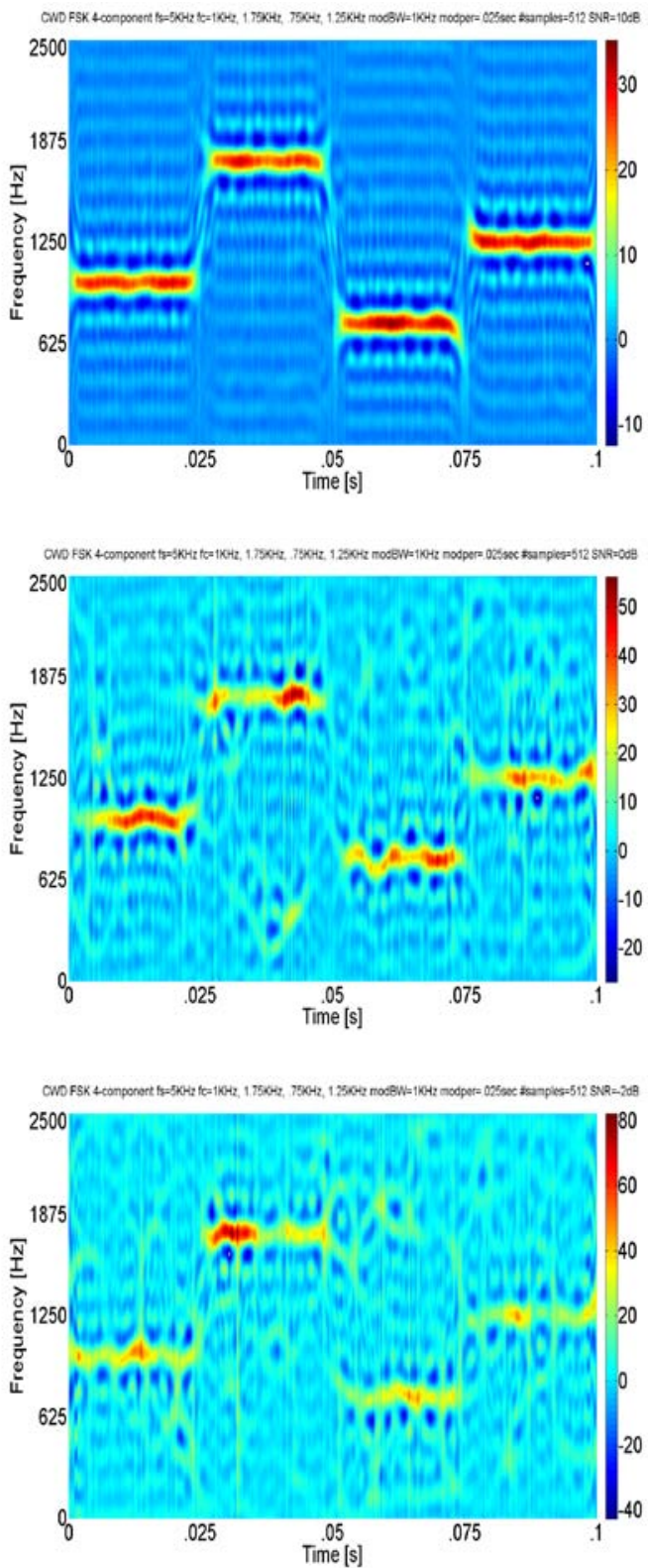

Figure 8: Comparative plots of the 4-component frequency hopping low probability of intercept radar signals (WVD (left-hand side) vs. CWD (right-hand side)). The SNR for the top row is $10 \mathrm{~dB}$, for the middle row is $0 \mathrm{~dB}$, and for the bottom row is $-2 \mathrm{~dB}$. The WVD signals are more localized ('thinner') than the CWD signals. However, the WVD does have a cross-term half-way between each signal, which, to the untrained eye, could be misinterpreted as a 'crossterm false positive' (the 6 blue 'false signals') - the more so as the SNR gets lower.

\section{Discussion}

This section will elaborate on the results from the previous section.

From Table 1, the WVD outperformed the CWD in average percent error: carrier frequency $(0.19 \%$ vs.
$0.62 \%)$, modulation bandwidth (5.97\% vs. $17.92 \%)$, and modulation period (17.01\% vs. $17.05 \%)$-and in average: time-frequency localization-y (as a percent of $y$-axis) (2.04\% vs. $6.78 \%)$ and percent detection $(90.7 \%$ vs. $88.7 \%)$. These results are by and large a result of the WVD signal being much more localized signal than the 
CWD signal. The CWD's 'thicker' signal is a result of its cross-term reduction - at the expense of signal localization.

The CWD outperformed the WVD in average: plot time (10.16s vs. 6382s) and lowest detectable SNR $(-2.2 \mathrm{db}$ vs. $-2.0 \mathrm{db})$. The combination of the CWD's reduction of cross-term interference along with the WVD being very computationally complex [MIL02] are the grounds for the CWD's better plot time. In addition, lowest detectable SNR is based on visual detection in the Time-Frequency representation. Figures 8 and 9 show that, for the WVD plots, as the SNR gets lower, it gets more difficult to distinguish between the actual signals and the cross-term interference. However, for the CWD plots there is no cross-term interference to confuse with the actual signals, making the CWD signals, though not as localized, easier to detect than the WVD signals- at these lower SNRs.

The WVD might be used in a scenario where you need good signal localization in a fairly low SNR environment, without tight time constraints. The CWD might be used in a scenario where a short plot time is necessary, and where signal localization is not an issue. Such a scenario might be a 'quick and dirty' check to see if a signal is present, without precise extraction of its parameters.

\section{Conclusions}

Digital intercept receivers, whose main job is to detect and extract parameters from low probability of intercept radar signals, are currently moving away from Fourier-based analysis and towards classical timefrequency analysis techniques, such as the WVD and the CWD, for the purpose of analyzing low probability of intercept radar signals. Based on the research performed for this paper (the novel direct comparison of the WVD versus the CWD for the signal analysis of low probability of intercept frequency hopping radar signals) it was shown that the WVD by and large outperformed the CWD for analyzing these low probability of intercept radar signals - for reasons brought out in the discussion section above. More accurate characterization metrics could well translate into saved equipment and lives.

Future plans include analysis of an additional low probability of intercept radar waveform (triangular modulated FMCW), again using the WVD and the CWD as time-frequency analysis techniques.

\section{References Références Referencias}

1. [ADA04] Adamy, D., EW 102: A Second Course in Electronic Warfare. Artech House, Norwood, MA, 2004

2. [AMS09] Anjaneyulu, L., Murthy, N., Sarma, N., Identification of LPI Radar Signal Modulation using Bi-coherence Analysis and Artificial Neural Networks
Techniques. NCC 2009, IIT Guwahati, pp. 19-22, January 16-18, 2009.

3. [ANJ09] Anjaneyulu, L., Murthy, N., Sarma, N., A Novel Method for Recognition of Modulation Code of LPI Radar Signals. International Journal of Recent Trends in Engineering, Vol. 1, No. 3, pp. 176-180, May 2009.

4. [AUG96] Auger, F., Flandrin, P., Goncalves, P., Lemoine, O., Time-Frequency Toolbox Users Manual. Centre National de la Recherche Scientifique and Rice University, 1996.

5. [BOA03] Boashash, B., Time Frequency Signal Analysis and Processing: A Comprehensive Reference. Elsevier, Oxford, England, 2003.

6. [CHO89] Choi, H., Williams, W., Improved TimeFrequency Representation of Multicomponent Signals Using Exponential Kernels. IEEE Transactions on Acoustics, Speech, and Signal Processing. Vol. 37, pp. 862-871, June 1989.

7. [GUL07] Gulum, T., Autonomous Non-Linear Classifications of LPI Radar Signal Modulations. Thesis, Naval Postgraduate School, Monterey, CA, 2007.

8. [GUL08] Gulum, T., Pace, P., Cristi, R., Extraction of Polyphase Radar Modulation Parameters Using a Wigner-Ville Distribution-Radon Transform. IEEE International Conference on Acoustics, Speech, and Signal Processing, Las Vegas, NV, April 2008.

9. [HAN00] Han, S., Hong, H., Seo, D., Choi, J., Target Position Extraction Based on Instantaneous Frequency Estimation in a Fixed-Reticle Seeker. Opt. Eng., Vol. 39, pp. 2568-2573, September 2000.

10. [HLA92] Hlawatsch, F., Boudreaux-Bartels, G.F., Linear and Quadratic Time-Frequency Signal Representations. IEEE Signal Processing Mag., Vol. 9, No. 2, pp. 21-67, April 1992.

11. [LIX08] Li, X., Bi, G., A New Reassigned TimeFrequency Representation. $16^{\text {th }}$ European Signal Processing Conference, Lausanne, Switzerland, pp. 1-4, August 25-29, 2008.

12. [LIY03] Li, Y., Xiao, $X .$, Recursive Filtering Radon-Ambiguity Transform Algorithm for Detecting Multi-LFM Signals. Journal of Electronics (China), Vol. 20, No. 3, pp. 161-166, May 2003.

13. [MIL02] Milne, P., Pace, P., Wigner Distribution Detection and Analysis of FMCW and P-4 Polyphase LPI Waveforms. Proceedings of ICASSP, Orlando, FL, pp. 3944-3947, 2002.

14. [OZD03] Ozdemir, A., Time-Frequency Component Analyzer. Dissertation, Bilkent University, Ankara, Turkey, Sept. 2003. 
15. [PAC09] Pace, P., Detecting and Classifying Low Probability of Intercept Radar. Artech House, Norwood, MA, 2009.

16. [PAP94] Papandreou, A., Boudreaux-Bartels, G.F., Kay, S., Detection and Estimation of Generalized Chirps Using Time-Frequency Representations. 1994 Conference Record of the Twenty-Eighth Asilomar Conference on Signals, Systems and Computers, pp. 50-54, 1994.

17. [QIA02] Qian, S., Introduction To TimeFrequency and Wavelet Transforms. Prentice Hall, Upper River, NJ, 2002.

18. [RAN01] Rangayyan, R., Krishnan, S., Feature Identification in the Time-Frequency Plane by Using the Hough-Radon Transform. Pattern Recognition, Vol. 34, pp. 1147-1158, 2001.

19. [STE96] Stephens, J., Advances in Signal Processing Technology for Electronic Warfare. IEEE AES Systems Magazine, pp. 31-38, November 1996.

20. [UPP08] Upperman, T., ELINT Signal Processing Using Choi-Williams Distribution on Reconfigurable Computers for Detection and Classification of LPI Emitters. Thesis, Naval Postgraduate School, Monterey, CA, March 2008.

21. [WEI03] Wei, G., Wu, S., Mao, E., Analysis of Multicomponent LFM Signals Using TimeFrequency and The Gray-Scale Inverse Hough Transform. IEEE Workshop on Statistical Signal Processing, pp. 190-193, September 28 - October 1, 2003.

22. [WIL06] Wiley, R., ELINT: The Interception and Analysis of Radar Signals. Artech House, Norwood, MA, 2006.

23. [WIL92] Williams, W., Jeong, J., Reduced Interference Time-Frequency Distributions. TimeFrequency Signal Analysis: Methods and Applications (B. Boashash, ed.), LongmanCheshire/Wiley, Melbourne/N.Y., 1992.

24. [XIA99] Xia, X., Chen, V., A Quantitative SNR Analysis for the Pseudo Wigner-Ville Distribution. IEEE Transactions on Signal Processing, Vol. 47, No. 10, pp. 2891-2894, October, 1999. 